\title{
STABILITY AND RESOLUTION OF NON-LINEAR DYNAMIC SYSTEMS CONTAINING TIME DELAYS
}

\author{
NGUYEN NHAT LE \\ Polytechnical University of Hanoi
}

\section{§1. INTRODUTION}

The stability and the vibration for non-linear dynamic system with time delay received considerable attention [1-6].

This study is concerned with the stability and the resolution of higher-dimensional systems containing several time delays. Two exemples are presented to illustrate the method.

\section{§2. FORMULATION OF PROBLEM}

\subsection{Definitions}

Let's consider the following $n$ th-order system containing two time delays:

$$
\dot{X}=f\left[X(t), X\left(t-\tau_{1}\right), X\left(t-\tau_{2}\right)\right]
$$

where $X$ and $f$ are $n$-dimensional column vectors; $\dot{X}$ denotes time derivative of $X ; \tau_{1}$ and $\tau_{2}$ are constants; $\left(\tau_{2}>\tau_{1}\right)$.

The origine is the steady state: $f(0,0,0)=0$.

The initial conditions are given by:

$$
X=g(t) \text { for } \quad-\tau_{2} \leq t \leq 0
$$

For any continuous initial function $g(t)$, assume that, $\phi(t, g)$ is a unique and continuous solution of (1).

For time delay systems, we have the following definitions [3]:

- The norme of state vector:

$$
\rho[X(t)]=\sup \left|x_{j}(t-\tau)\right| \text { for } 0 \leq \tau \leq \tau_{2} ; \quad j=1, \ldots, n
$$
that:

- The equilibrium of equation (1) is said to be stable if for every $\varepsilon>0$ there is a $\delta(\varepsilon)>0$ such implies that

$$
\rho[g(t)]<\varepsilon \text { for }-\tau_{2} \leq t \leq 0
$$

$$
\rho[\phi(t, g)]<\varepsilon \text { for } t>0
$$

The equilibrium of equation (1) is said to be asymptotically stable if

(i) it is stable

(ii) there is a $\delta_{1}(\varepsilon)>0$ such that for all $\rho[g(t)]<\delta_{1}$, then

$$
\lim \rho[\phi(t, g)] \rightarrow 0 \quad \text { as } t \rightarrow \infty
$$


- Let $R$ be a closed and bounded region in the $n$-dimensional state space and assume that $R$ contains the origin then $R$ is a region of stability for equation (1) if for all

$$
g(t) \in R \text { for }-\tau_{2} \leq t \leq 0
$$

then

$$
\phi(t, g) \in R \text { for } t>0 .
$$

- The region $R$ is called the region of asymptotic stability for the systems in equations (1), if for all $g(t) \in R$ and for $-\tau_{2} \leq t \leq 0$ :

(i) $R$ is a region of stability

(ii) $\lim \rho[\phi(t, g)] \rightarrow 0$ as $t \rightarrow \infty$

\subsection{Formulation of problem}

For time delay system (1), the problem of stability and resolution is formulated as following:

Determine the region of stability for several values of time delays and search a convenient algorithm to solve the system of non-linear differential equations containing time delays (1) with initial conditions (2)

\section{§3. CONSTRUCTION OF REGION OF STABILITY}

Let's return to the system (1) and denote $F$ the vector equation corresponding system without delay (i.e. $\tau_{1}-\tau_{2}=0$ ).

$$
\dot{X}=F(X) ; \quad F(0)=0 .
$$

For stability of time delays systems we can use Liapunov's second method, that has been developed for non-lineare system with time delay [3]; [4].

\subsection{Construction of Liapunov's function}

For many stability problems, a Liapunov's function has the type:

$$
V(X)=F^{T} \dot{A} F
$$

where $F$ is the derivative vector and $A$ is a positive-definite matrix. For the time delay system (1) the Liapunov's function can take type $V=F^{T} F$.

Suppose that $V(X)$ is a positive-definite function such that $V(X)=K=$ const definies a. closed surface.

Let $S$ be the boundary of a closed region $R$ that contains the origin (Fig. 1).

Assume that $V(X)$ has continuous first partial derivatives on $S$ and the time derivative

$$
\dot{V}(X)<0 \text { for all } X \in S .
$$

Thus at each point on the boundary $S$ of a region $R$, the derivative vector $f$ is allways directed into $R$. Since

$$
\dot{V}=\nabla V \cdot f\left[X(t), X\left(t-\tau_{1}\right), X\left(t-\tau_{2}\right)\right] ; \quad \tau_{2}>\tau_{1}
$$

at each point on the boundary, $\dot{V}$ depends on the past state $X\left(t-\tau_{1}\right), X\left(t-\tau_{2}\right)$. We must now construct the regions of possible past states [4].

3,2. Regions of possible past states

If upper bounds on the time derivatives of the state variables are known or can be estimated; i.e.

$$
f_{j \max }=\max \left|f_{j}\right|
$$


then the region $\Omega_{k}$ is defined as follows for any current state $X^{*}$ :

$$
\Omega_{k}=\left\{X:\left|x_{j}-x_{j}^{*}\right| \leq f_{j \max } \tau_{k} ; \quad j=1, n ; \quad k=1,2\right\}
$$

and region of possible past states:

$$
\Omega_{\ell}^{\prime}=\Omega_{k} \cap R \quad \text { for } \quad k=1,2 .
$$

\subsection{Sufficient condition for a region of stability}

Let's denote:

$$
\dot{V}_{M}\left(X^{*}\right)=\max \dot{V}(X) \quad \text { for } \quad X=X^{*} ; \quad X\left(t-\tau_{k}\right) \in \Omega_{k}^{\prime}
$$

and $R_{0}=\{X:\|X\|<r\}$ for some $r>0$

Assume that in $R_{0}$, the function $V(X)$ is positive definite and $V(X)=K$ defines a closed surface $S$ and bounded region $V(X)<K$ as $R \subset R_{0}$ then $R$ is a region of stability for time delay system (1) if:

$$
\dot{V}_{M}\left(X^{*}\right)<0 \text { for all } X^{*} \in S
$$

\section{Computational algorithm}

Using above sufficient condition, we can determine the region of stability for time delay system in the following manner:

(1) Select a Liapunov's function

(2) Select an initial value for the contour parameter $K$

(3) Calculate $f_{1 \max }, f_{2 \max }$

(4) Evaluate $V(X)$ along the curve $V(X)=K$

(5) Increase the value of $K$ and repeat steps (3) and (4) until the maximum region of stability is obtained.

In the partie of application is constructed a region of stability for a concrete example.

\section{§4. NUMERICAL RESOLUTION FOR SYSTEM OF NON-LINEAR DIFFERENTIAL EQUATIONS CONTAINING TIME DELAYS}

Using numerical methods to resolve the non-linear differential equations with time delays we can apply the algorithms of ordinary differential equations without time delay, howeve a procedure to time delays must be added as shown in fig. 2.

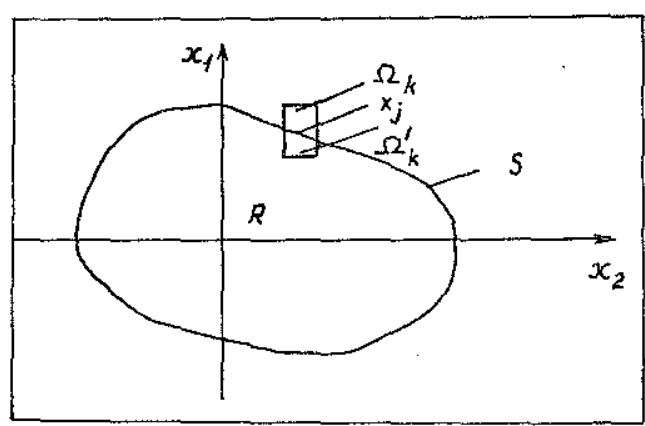

Fig. 1

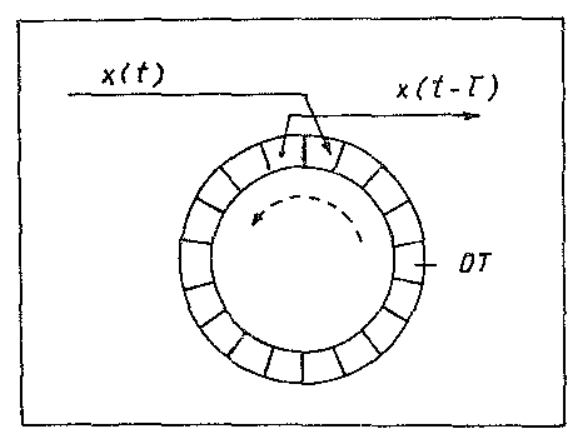

Fig. 2

where $\tau$ - time delay, $D T$ - time step

Also, we can use the blocs creating time delays in the computer programme of simulations. 


\section{EXEMPLES}

\subsection{Exemple 1}

System of differential equations with time delays is given:

$$
\begin{aligned}
& \dot{x}_{1}=1,2-2,1 x_{1}(t)-0,4 x_{1}\left(t-\tau_{1}\right)=f_{1} \\
& \dot{x}_{2}=-1,2-2 x_{1}(t)-4 x_{2}\left(t-\tau_{2}\right)=f_{2}
\end{aligned}
$$

The corresponding system without time delay:

$$
\begin{aligned}
& \dot{x}_{1}=1,2-2,5 x_{1}(t)=F_{1} \\
& \dot{x}_{2}=-1,2-2 x_{1}(t)-4 x_{2}(t)=F_{2}
\end{aligned}
$$

The Liapunov's function:

$$
V(X)=F_{1}^{2}+F_{2}^{2}
$$

The contour of $V(X)=K=$ const is shown in fig. 3

Calculating regions of possible past states $\left(f_{\mathrm{t} \max }, f_{2 \mathrm{max}}\right)$ and evaluating the derivative $\dot{V}(X)$ along those contours we will obtain the region of stability.

The behaviours of states $x_{1}(t), x_{1}\left(t-\tau_{1}\right), x_{2}(t)$ and $x_{2}\left(t-\tau_{2}\right)$ are shown in fig. 4

The influence of time delays is shown in fig. 5 where

curve 1 and $2: x_{1}(t)$ and $x_{2}(t)$ for system without delay, delay.

curve 3 and $4: x_{1}(t)$ and $x_{2}(t)$ for system with

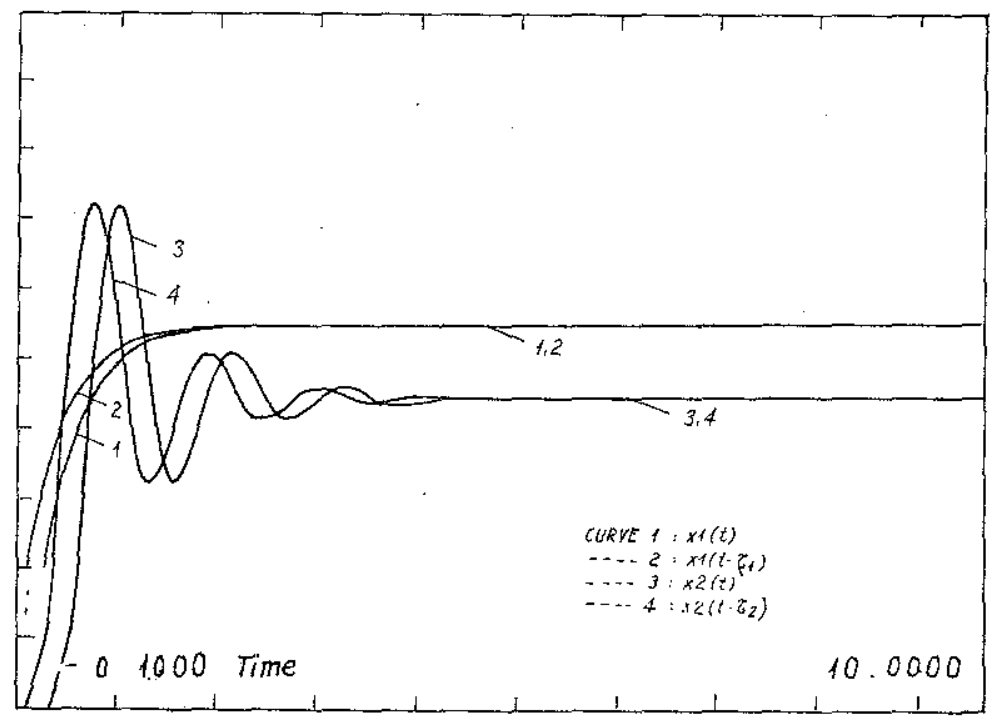

Fig. 4 


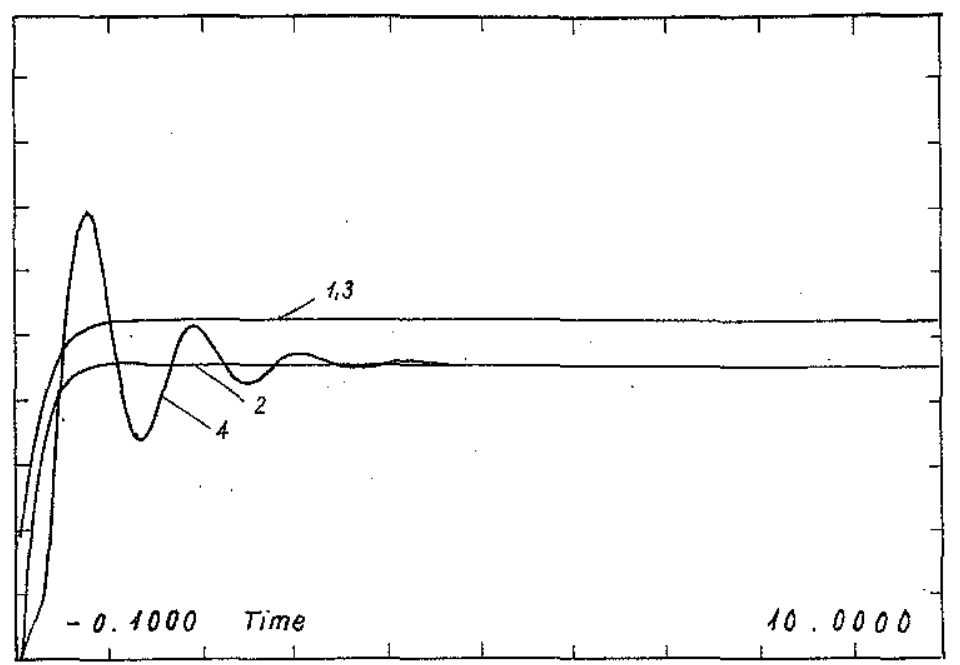

Fig. 5

Thus, in this case, the time delay system has the vibrational character of state variable $x_{2}(t)$.

5.2. Example 2

Differential equation with time delay:

$$
\begin{aligned}
\dot{X} & =\varepsilon X^{2}(t-\pi) \cos t \\
X(0) & =0,25 ; \quad \varepsilon \leq 0,2
\end{aligned}
$$

This differential equation has an analytical solution in the first approximation [1]:

$$
X=0,25+0,0125 \sin t .
$$

The corresponding behaviour of $X(t)$ showns in fig. 6

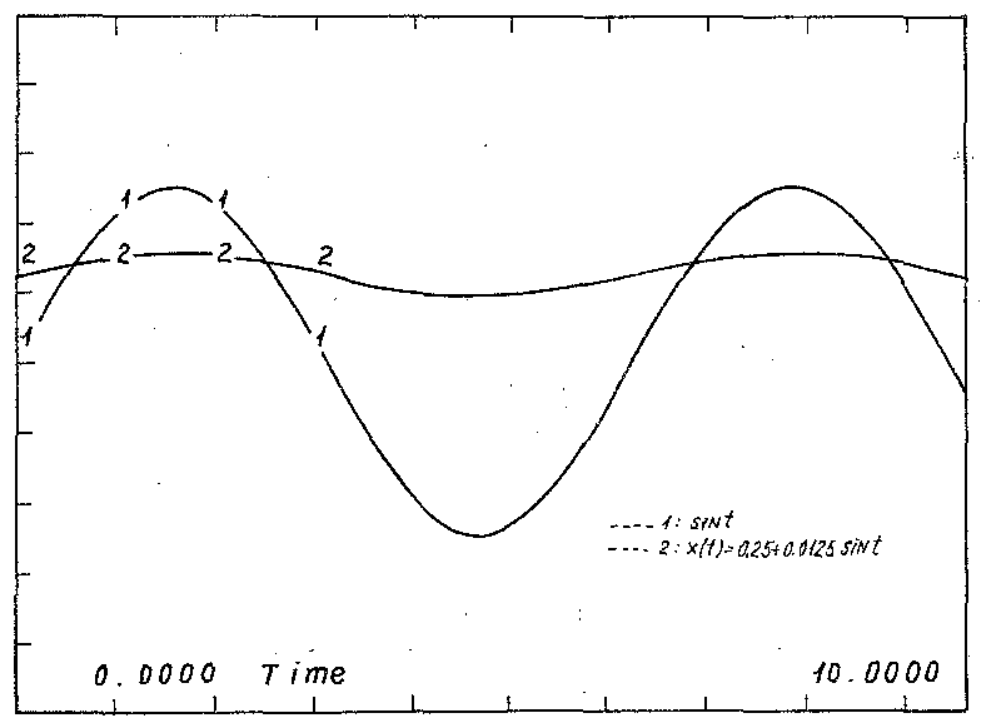

Fig. 6

Using computational algorithm presented in part IV, we receive the numerical results and the behaviour of state $X(t)$ as shown in fig. 7 


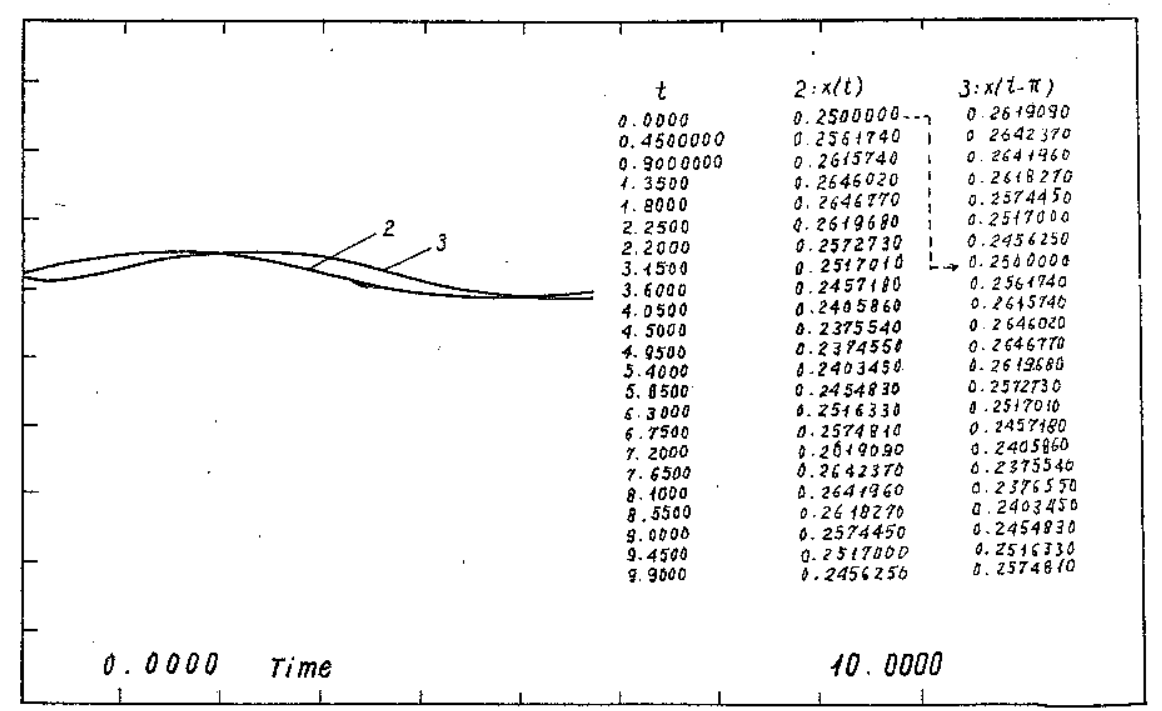

Fig. 7

Comparing curve 2 in fig. 6 and that in fig. 7 , we see that in this case, the numerical results are correspondent to the analytical results.

\section{CONCLUSION}

This study has research the region of stability and computer resolution for non-linear systems containing time delays, two exemples are consided and the numerical results are compared with the analytical results.

This publication is completed with financial support from the National Basis Research Program in Natural Sciences.

\section{REFERENCES}

1. Mitropolskii Yu. and Martuniuk. Periodic and quasi-periodic oscillations of systems with delay, Moscow, 1979.

2. Rubanic V. P. Oscillations of quasi-periodic system with delay, Moscow, 1969.

3. Krasovskii N. N. Some problems of stability of Motion, Moscow, 1959.

4. Dale E. Sorborg and Ernest F. Johnson. Construction of regions of stability for non-linear systems containing time delays. Int. J. Control, 1970, Vol. 12, No. 6.

5. Nguyen Dong Anh, Nguyen Tien Khiem. Oscillations in the first order systems with delay. Journal of Mechanics, No 1, No 2, No 3, 1990.

6. Nguyen Nhat Le. Optimal Control of systems with time delays. Journal of Mechanics, Vol. 14, No $3,1992$.

Received April 14, 1999

\section{SỰ ỔN ĐỊNH VÀ GIẢI CÁC Hệ ĐộNG LỰC PHI TUYẾN CHỨA THỜI GIAN CHẬM}

Xét miền ổn định và phương pháp số để giải hệ phương trình vi phân phi tuyến cấp cao chứa thời gian chậm.

Đã xét hai thí dụ cụ thể. Kết quả được so sánh với kết quả giải tích của xấp xỉ thứ nhất. 